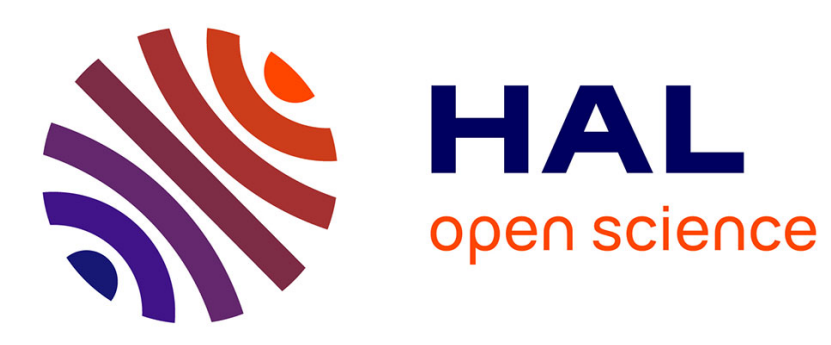

\title{
Structure cristalline de la L-glycyl-L-leucyl-L-phénylalanine hémihydraté
} Jean Delettré, Jean Berthou, Alain Lifchitz, Pierre Jollès

\section{To cite this version:}

Jean Delettré, Jean Berthou, Alain Lifchitz, Pierre Jollès. Structure cristalline de la L-glycyl-L-leucylL-phénylalanine hémihydraté. Acta Crystallographica Section C : Structural Chemistry [2014-..], 1988, 44, pp.902-904. 10.1107/S0108270188000952 . hal-00015165

\section{HAL Id: hal-00015165 https://hal.science/hal-00015165}

Submitted on 24 Feb 2006

HAL is a multi-disciplinary open access archive for the deposit and dissemination of scientific research documents, whether they are published or not. The documents may come from teaching and research institutions in France or abroad, or from public or private research centers.
L'archive ouverte pluridisciplinaire HAL, est destinée au dépôt et à la diffusion de documents scientifiques de niveau recherche, publiés ou non, émanant des établissements d'enseignement et de recherche français ou étrangers, des laboratoires publics ou privés. 
Acta Cryst. (1988). C44, 902-904

\title{
Structure Cristalline de la L-Glycyl-L-leucyl-L-phenylalanine Hémihydraté
}

\author{
Par Jean Delettré, Jean Berthou et Alain Lifchitz
}

Laboratoire de Minéralogie et Cristallographie (LA CNRS n $n^{0}$ 09), Université Pierre et Marie Curie, 75231 Paris CEDEX 05, France

et Pierre Jollès

Laboratoire des Protéines (UA CNRS no 1188), Université Paris V, 45 rue des Saints Pères, 75270 Paris CEDEX 06, France

(Reçu le 23 septembre 1987, accepté le 20 janvier 1988)

\begin{abstract}
C}_{17} \mathrm{H}_{25} \mathrm{~N}_{3} \mathrm{O}_{4} \cdot \frac{1}{2} \mathrm{H}_{2} \mathrm{O}, M_{r}=344.4$, monoclinic, $1.202 \mathrm{Mg} \mathrm{m}^{-3}$, room temperature, final $R=0.048$ and $B 2, a=18.299(6), b=18.781(6), c=5.917$ (3) $\AA, w R=0.048$ for all (1470) reflections. The molecules $\gamma=110.65(3)^{\circ}, V=1902.9(10) \AA^{3}, Z=4, \mathrm{Cu} K \alpha$, are stacked in layers along the $a$ axis. There are five $\lambda=1.54178 \AA, \mu=0.73 \mathrm{~mm}^{-1}, F(000)=740, D_{x}=$ intermolecular hydrogen bonds.

0108-2701/88/050902-03\$03.00 c) 1988 International Union of Crystallography
\end{abstract}


Introduction. De nombreux peptides se sont révélés comme de puissants agents immunomodulateurs. Cette découverte a suscité un intérêt considérable quant à leur utilisation en thérapeutique humaine. Pour une revue générale, voir Werner, Floc'h, Migliore-Samour \& Jollès (1986). Certains sont d'origine exogène, soit microbienne comme le muramyl dipeptide (MDP), soit fongique comme la cyclosporine. A partir de ces substances on a pu synthétiser des dérivés ou des analogues, tous actifs, comme des lipopeptides (Migliore-Samour, Bouchaudon, Floc'h, Zerial, Ninet, Werner \& Jollès, 1980) ou des dérivés du MDP (Adam \& Lederer, 1984).

Il existe des peptides d'origine endogène possédant les mêmes propriétés comme les hormones thymiques (Bach, 1984) et la tuftsine (Najjar \& Fridkin, 1983). Il a été montré (Jollès, Parker, Floc'h, Migliore, Alliel, Zerial \& Werner, 1982) que des fragments de la caséine humaine, fraction proteique principale du lait maternel, possédaient, eux-aussi, des propriétés stimulantes. De cette caséine, de courts peptides actifs, ont pu être isolés et caractérisés comme l'hexapeptide Val-Glu-Pro-IlePro-Tyr (Parker et al., 1984). Mieux encore, des tripeptides de même origine possèdent cette propriété (Berthou, Migliore-Samour, Lifchitz, Delettré, Floc'h \& Jollès, 1987).

C'est dans ce contexte que nous proposons l'étude du tripeptide naturel L-glycyl-leucyl-phenylalanine (GLP) afin de rechercher une éventuelle influence conformationnelle sur son activité.

Partie expérimentale. Le cristal, 1,5 $\times 0,3 \times 0,1 \mathrm{~mm}$, a été obtenu sous forme d'un hémihydrate par évaporation lente (six semaines) d'une solution méthanol-eau (1:3). Mesures: diffractomètre Philips PW 1100, monochromateur graphite, température ambiante; mesure des intensités par 'flying stepscan'; 1470 réflexions mesurées; réflexions de contrôle $2 \overline{1} 1,11 \overline{1}$, $\overline{2} 20$, variations maximales de $0,4 \%$ autour de la valeur moyenne; $-20 \leq h \leq 18, \quad-20 \leq k \leq 9, \quad 0 \leq l \leq 6$; valeurs moyennées pour $|k| \geq 10$; paramètres cristallins obtenus à partir de 25 réflexions $5<\theta<20^{\circ}$.

Corrections de Lorentz-polarisation, pas de correction d'absorption. $[(\sin \theta) / \lambda]_{\max }=0,549 \AA^{-1}$; résolution par méthodes directes: programme MULTAN (Germain, Main \& Woolfson, 1971), facteurs de diffusion atomique (Cromer \& Mann, 1968). Affinement en matrice complète: programme AFFINE (Delettré, Mornon \& Lepicard, 1980) converge jusqu'à $R=$ $0,048, w R=0.048$; minimisation de $\sum w\left(\left|F_{o}\right|-\left|F_{c}\right|\right)^{2}$ avec $w=1 / \sigma^{2}$; atomes $H$ placés sur section de Fourier-différence et affinés; coefficients d'agitation thermique anisotrope pour tous les atomes sauf $\mathrm{H}$; résidu de densité électronique sur sections de Fourierdifference finales $0,46 \mathrm{e} \AA^{-3} ;(\Delta / \sigma)_{\max }$ pour les $x, y, z$ des C, O: 0,6.
Tableau 1. Coordonnées atomiques fractionnaires $\left(\times 10^{4}\right)$ avec leurs écarts-type entre parenthèses et facteurs d'agitation thermique équivalents

\begin{tabular}{|c|c|c|c|c|}
\hline & & $\frac{4}{3} \sum_{i} \sum_{j} \beta$ & & \\
\hline Glycyl & $x$ & $y$ & $z$ & $B_{\dot{e q}}\left(\AA^{2}\right)$ \\
\hline $\begin{array}{l}\mathrm{N} \\
\mathrm{C} \alpha \\
\mathrm{C}^{\prime} \\
\mathrm{O}\end{array}$ & $\begin{array}{l}-3060(2) \\
-3146(2) \\
-3040(2) \\
-2816(2)\end{array}$ & $\begin{array}{r}46(2) \\
800(2) \\
1224(2) \\
1010(2)\end{array}$ & $\begin{array}{l}2081(24) \\
1710(10) \\
3948(10) \\
5663(8)\end{array}$ & $\begin{array}{l}3.7(2) \\
3.9(2) \\
3.5(2) \\
4.7(1)\end{array}$ \\
\hline $\begin{array}{l}\text { Leucyl } \\
\mathrm{N} \\
\mathrm{C} \alpha \\
\mathrm{C}^{\prime} \\
\mathrm{O} \\
\mathrm{C} \beta \\
\mathrm{C} \gamma \\
\mathrm{C} \delta(1) \\
\mathrm{C} \delta(2)\end{array}$ & $\begin{array}{l}-3218(2) \\
-3109(2) \\
-3728(2) \\
-3561(2) \\
-3089(2) \\
-2391(3) \\
-2540(4) \\
-1643(3)\end{array}$ & $\begin{array}{l}1869(2) \\
2371(2) \\
2090(2) \\
2275(2) \\
3155(2) \\
3584(2) \\
4223(3) \\
3929(4)\end{array}$ & $\begin{array}{l}3809(8) \\
5725(10) \\
7543(9) \\
9520(8) \\
4882(10) \\
3394(12) \\
2100(14) \\
4823(19)\end{array}$ & $\begin{array}{r}3.5(1) \\
3.4(2) \\
3.4(2) \\
4.8(1) \\
4.0(2) \\
5.2(2) \\
8.1(3) \\
10.5(4)\end{array}$ \\
\hline $\begin{array}{l}\text { Phenyl } \\
\mathrm{N} \\
\mathrm{C} \alpha \\
\mathrm{C}^{\prime} \\
\mathrm{O}^{\prime} \\
\mathrm{O}^{\prime \prime} \\
\mathrm{C} \beta \\
\mathrm{C} \gamma \\
\mathrm{C} \delta(1) \\
\mathrm{C} \delta(2) \\
\mathrm{C} \delta(1) \\
\mathrm{C} \varepsilon(2) \\
\mathrm{C} \zeta\end{array}$ & $\begin{array}{l}-4446(2) \\
-5126(2) \\
-5837(2) \\
-5734(2) \\
-6463(1) \\
-5307(2) \\
-5292(2) \\
-5829(4) \\
-4734(3) \\
-5811(6) \\
-4700(4) \\
-5257(5)\end{array}$ & $\begin{array}{r}1684(2) \\
1417(2) \\
904(2) \\
822(2) \\
636(2) \\
2073(3) \\
2703(2) \\
2589(3) \\
3410(3) \\
3141(4) \\
3992(3) \\
3816(4)\end{array}$ & $\begin{array}{l}6864(8) \\
8325(10) \\
6943(10) \\
4873(8) \\
7970(8) \\
9492(10) \\
7913(12) \\
6196(15) \\
8066(17) \\
4639(18) \\
6431(21) \\
4775(19)\end{array}$ & $\begin{array}{r}3.9(1) \\
3.8(2) \\
3.8(2) \\
4.5(1) \\
4.7(1) \\
4.7(2) \\
4.8(2) \\
8.9(4) \\
7.9(3) \\
13.1(6) \\
10.9(5) \\
11.3(5)\end{array}$ \\
\hline $\begin{array}{l}\text { Solvan } \\
\text { Wat }\end{array}$ & 0 & 0 & $7433(12)$ & $6,8(3)$ \\
\hline
\end{tabular}

Discussion. Les coordonnées atomiques sont reportées dans le Tableau 1,* les longueurs de liaison, angles de valence et angles de torsion dans le Tableau 2. La Fig. 1 montre la projection selon l'axe $c$.

Nous sommes en présence d'un zwitterion tout à fait caractéristique. Notons la ségrégation des groupes hydrophobes des chaines latérales de la phénylalanine et de la leucine qui délimitent un canal parallèle à l'axe $a$ de diamètre environ $4 \AA$. Un tel regroupement a déjà été observé (Prangé \& Pascard, 1979). Il en résulte une grande agitation de ces chaînes. Ce phénomène est courant dans de tels composés (Précigoux, Geoffre \& Ouvrard, 1986). Notons les longueurs de liaison $\mathrm{C} \varepsilon(1)-\mathrm{C} \zeta, \quad 1,32$ et $\mathrm{C} \delta(2)-\mathrm{C} \varepsilon(2), \quad 1,45 \AA$ dont l'interprétation ne semble pas immédiate. Les liaisons hydrogène rencontrées sont tout à fait classique, remarquons toutefois l'absence de telles liaisons sur $\mathrm{O}$ (Gly) et N (Phe), voir Tableau 3.

Les liaisons peptidiques sont bien en conformation trans, les groupes peptidiques restent plans $(\omega=176$ et $175^{\circ}$ ). Les angles $\varphi$ et $\psi$ sont tout à fait normaux mais l'extrémité phénylalanine est en conformation étendue $\left(\varphi=-174,9^{\circ}\right)$.

\footnotetext{
* Les listes des facteurs de structure, des paramètres d'agitation thermique anisotrope, des angles de valence, et des coordonnées des atomes d'hydrogène, ont été déposées au dépôt d'archives de la British Library Document Supply Centre (Supplementary Pub. lication No. SUP 44711: 14 pp.). On peut en obtenir des copies en s'adressant à: The Executive Secretary, International Union of Crystallography, 5 Abbey Square, Chester CH1 2HU, Angleterre.
} 
Tableau 2. Longueurs de liaison $(\AA)$, angles de valence $\left.{ }^{\circ}\right)$ et angles de torsion $\left(^{\circ}\right)$, les déviations standards étant données entre parenthèses

Angles de torsion en accord avec les normes de la IUPAC-IUB Commission on Biochemical Nomenclature (1970).

$\begin{array}{ll}\text { Glycyl } & \\ \mathrm{N}-\mathrm{C} \alpha & \\ \mathrm{C} \alpha-\mathrm{C}^{\prime} & 1,496(6) \\ \mathrm{C}^{\prime}-\mathrm{O} & 1,522(8) \\ \mathrm{C}^{\prime}-\mathrm{N} & 1,215(7) \\ \text { Leucyl } & 1,362(6) \\ \mathrm{N}-\mathrm{C} \alpha & \\ \mathrm{C} \alpha-\mathrm{C}^{\prime} & \\ \mathrm{C}^{\prime}-\mathrm{O} & 1,444(7) \\ \mathrm{C} \alpha-\mathrm{C} \beta & 1,516(7) \\ \mathrm{C} \beta-\mathrm{C} \gamma & 1,228(7) \\ \mathrm{C} \gamma-\mathrm{C} \delta(1) & 1,543(6) \\ \mathrm{C} \gamma-\mathrm{C} \delta(2) & 1,526(7) \\ \mathrm{C}^{\prime}-\mathrm{N} & 1,526(9) \\ & 1,544(9)\end{array}$

Phénylalanine

$\begin{array}{ll}\mathrm{N}-\mathrm{C} a & 1,452(6) \\ \mathrm{C} a-\mathrm{C}^{\prime} & 1,550(6) \\ \mathrm{C}^{\prime}-\mathrm{O}^{\prime \prime} & 1,236(5) \\ \mathrm{C}^{\prime}-\mathrm{O}^{\prime} & 1,257(8) \\ \mathrm{C} \alpha-\mathrm{C} \beta & 1,546(7) \\ \mathrm{C} \beta-\mathrm{C} \gamma & 1,500(8) \\ \mathrm{C} \gamma-\mathrm{C} \delta(1) & 1,378(10) \\ \mathrm{C} \gamma-\mathrm{C} \delta(2) & 1,363(6) \\ \mathrm{C} \delta(1)-\mathrm{C} \varepsilon(1) & 1,379(12) \\ \mathrm{C} \delta(2)-\mathrm{C} \varepsilon(2) & 1,445(12) \\ \mathrm{C} \varepsilon(1)-\mathrm{C} \zeta & 1,317(9) \\ \mathrm{C} \varepsilon(2)-\mathrm{C} \zeta & 1,367(14)\end{array}$

Angles dièdres du squelette $\left(^{\circ}\right)$ Glycyl

$$
\begin{array}{ll}
\psi & -171,4 \\
\omega & -176,2
\end{array}
$$

Leucyl

$$
\begin{array}{lr}
\varphi & -76,4 \\
\psi & -32,9 \\
\omega & -174,9
\end{array}
$$

Phenylalanine

$$
\varphi \quad-174,8
$$

$\begin{array}{ll}\mathrm{N}-\mathrm{C} \alpha-\mathrm{C}^{\prime} & 109,4(5) \\ \mathrm{C} \alpha-\mathrm{C}^{\prime}-\mathrm{O} & 123,4(4) \\ \mathrm{C} \alpha-\mathrm{C}^{\prime}-\mathrm{N} & 112,7(5) \\ \mathrm{O}-\mathrm{C}^{\prime}-\mathrm{N} & 123,9(5) \\ & \\ & \\ \mathrm{C}^{\prime}-\mathrm{N}-\mathrm{C} \alpha & 120,9(4) \\ \mathrm{N}-\mathrm{C} \alpha-\mathrm{C}^{\prime} & 114,5(3) \\ \mathrm{N}-\mathrm{C} \alpha-\mathrm{C} \beta & 108,8(4) \\ \mathrm{C}-\mathrm{C} \alpha-\mathrm{C} \beta & 109,0(4) \\ \mathrm{C} \alpha-\mathrm{C} \beta-\mathrm{C} \gamma & 115,1(4) \\ \mathrm{C} \beta-\mathrm{C} \gamma-\mathrm{C} \delta(1) & 110,9(5) \\ \mathrm{C} \beta-\mathrm{C} \gamma-\mathrm{C} \delta(2) & 111,0(6) \\ \mathrm{C} \delta(1)-\mathrm{C} \gamma-\mathrm{C} \delta(2) & 108,8(4) \\ \mathrm{C} \alpha-\mathrm{C}^{\prime}-\mathrm{O} & 119,8(3) \\ \mathrm{C} \alpha-\mathrm{C}^{\prime}-\mathrm{N} & 116,9(5) \\ \mathrm{O}-\mathrm{C}^{\prime}-\mathrm{N} & 123,2(4) \\ & \end{array}$

$\begin{array}{ll}\mathrm{C}^{\prime}-\mathrm{N}-\mathrm{C} \alpha & 124,9(5) \\ \mathrm{N}-\mathrm{C} \alpha-\mathrm{C}^{\prime} & 109,3(4) \\ \mathrm{N}-\mathrm{C} \alpha-\mathrm{C} \beta & 112,9(3) \\ \mathrm{C}^{\prime}-\mathrm{C} \alpha-\mathrm{C} \beta & 111,3(4) \\ \mathrm{C} \alpha-\mathrm{C} \beta-\mathrm{C} \gamma & 113,6(5) \\ \mathrm{C} \delta(1)-\mathrm{C} \gamma-\mathrm{C} \beta & 121,6(4) \\ \mathrm{C} \delta(2)-\mathrm{C} \gamma-\mathrm{C} \beta & 121,8(6) \\ \mathrm{C} \delta(1)-\mathrm{C} \gamma-\mathrm{C} \delta(2) & 116,6(6) \\ \mathrm{C} \gamma-\mathrm{C} \delta(1)-\mathrm{C} \varepsilon(1) & 123,4(5) \\ \mathrm{C} \gamma-\mathrm{C} \delta(2)-\mathrm{C} \varepsilon(2) & 120,7(7) \\ \mathrm{C} \delta(1)-\mathrm{C} \varepsilon(1)-\mathrm{C} \zeta & 119,3(9) \\ \mathrm{C} \delta(2)-\mathrm{C} \varepsilon(2)-\mathrm{C} \zeta & 117,9(5) \\ \mathrm{C} \varepsilon(1)-\mathrm{C} \zeta-\mathrm{C} \varepsilon(2) & 122,1(9) \\ \mathrm{O}^{\prime}-\mathrm{C}^{\prime}-\mathrm{C} \alpha & 117,4(4) \\ \mathrm{O}^{\prime \prime}-\mathrm{C}^{\prime}-\mathrm{C} \alpha & 116,6(5) \\ \mathrm{O}^{\prime \prime}-\mathrm{C}^{\prime}-\mathrm{O}^{\prime} & 125,9(4)\end{array}$

Angles dièdres des chaines latérales $\left(^{\circ}\right)$

$\mathrm{N}-\mathrm{C} \alpha-\mathrm{C} \beta-\mathrm{C} \gamma$ $\mathrm{C} \alpha-\mathrm{C} \beta-\mathrm{C} \gamma-\mathrm{C} \delta(1)$

$\mathrm{C} \alpha-\mathrm{C} \beta-\mathrm{C} \gamma-\mathrm{C} \delta(2)$

$$
\begin{array}{r}
-66,1 \\
163,3 \\
-75,7
\end{array}
$$

$\mathrm{N}-\mathrm{C} \alpha-\mathrm{C} \beta-\mathrm{C} \gamma$ $\mathrm{C} \alpha-\mathrm{C} \beta-\mathrm{C} \gamma-\mathrm{C} \delta(1)$ $\mathrm{C} \alpha-\mathrm{C} \beta-\mathrm{C} \gamma-\mathrm{C} \delta(2)$ $\mathrm{C} \beta-\mathrm{C} \gamma-\mathrm{C} \delta(1)-\mathrm{C} \varepsilon(1)$ $\mathrm{C} \beta-\mathrm{C} \gamma-\mathrm{C} \delta(2)-\mathrm{C} \varepsilon(2)$ $\mathrm{C} \gamma-\mathrm{C} \delta(1)-\mathrm{C} \varepsilon(1)-\mathrm{C} \zeta$ $\mathrm{C} \gamma-\mathrm{C} \delta(1)-\mathrm{C} \varepsilon(1)-\mathrm{C} \zeta$
$\mathrm{C} \gamma-\mathrm{C} \delta(2)-\mathrm{C} \varepsilon(2)-\mathrm{C} \zeta$ $\mathrm{C} \gamma-\mathrm{C} \delta(2)-\mathrm{C} \varepsilon(2)-\mathrm{C} \zeta$
$\mathrm{C} \delta(1)-\mathrm{C} \varepsilon(1)-\mathrm{C} \zeta-\mathrm{C} \varepsilon(2)$ $\mathrm{C} \delta(2)-\mathrm{C} \varepsilon(2)-\mathrm{C} \zeta-\mathrm{C} \varepsilon(1)$

$$
\begin{array}{r}
47,2 \\
65,7 \\
-112,5 \\
-176,5 \\
75,8 \\
-0,1 \\
1,7 \\
-0,8 \\
-0,0
\end{array}
$$

\begin{tabular}{|c|}
\hline $\mathrm{N}(\mathrm{Gly})(\mathrm{i}) \mathrm{H} \cdots \mathrm{O}^{\prime}(\mathrm{Phe})(\mathrm{v})$ \\
\hline $\mathrm{N}(\mathrm{Gly})(\mathrm{i}) \mathrm{H} \cdots \mathrm{O}^{\prime \prime}(\mathrm{Phe})(\mathrm{iii})$ \\
\hline $\mathrm{N}(\mathrm{Gly})(\mathrm{i}) \mathrm{H} \cdots \mathrm{O}^{\prime \prime}($ (Phe)(vi) \\
\hline $\mathrm{N}(\mathrm{Leu})(\mathrm{i}) \mathrm{H} \cdots \mathrm{O}(\mathrm{Leu})(\mathrm{ii})$ \\
\hline Wat(i)H $\cdots \mathrm{O}^{\prime}($ Phe)(iv) \\
\hline
\end{tabular}$$
0,0
$$

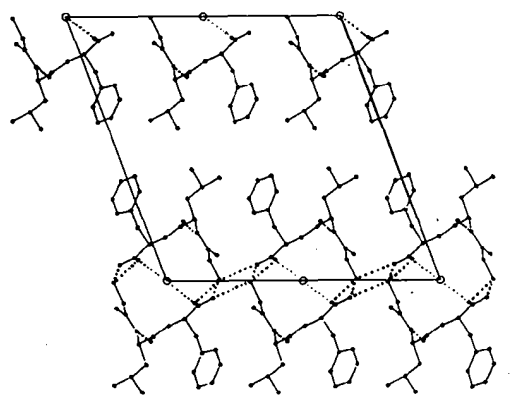

Fig. 1. Projection de l'arrangement moléculaire selon l'axe $c$. Les liaisons hydrogène sont représentées en pointillés.
Tableau 3. Liaisons hydrogène $(\AA)$

Les opérations de symétrie sont: (i) $x, y, z$; (ii) $x, y, 1+z$; (iii) $\frac{1}{2}+x, y, \frac{1}{2}+z$; (iv) $\frac{1}{2}+x, y,-\frac{1}{2}+z$; (v) $-1-x,-y, z$; (vi) $-1-x,-y, 1+z$.

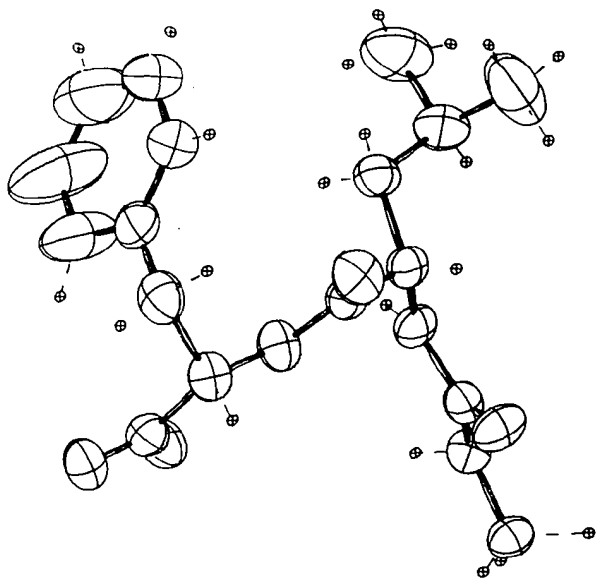

Fig. 2. Représentation des ellipsoïdes d'agitation thermique à $50 \%$ de probabilité.

Les régions hydrophiles, $\mathrm{COO}^{-}$et $\mathrm{NH}_{3}^{+}$, sont fédérées par une molécule d'eau. Le réseau de liaisons hydrogène est donné par le Tableau 3 ; il est constitué de cinq liaisons voisines de $2,8 \AA$.

La Fig. 2 représente les ellipsoïdes d'agitation thermique.

\section{Références}

AdAm, A. \& Lederer, E. (1984). Med. Res. Rev. 4, 111-152.

BACH, J. F. (1984). Dans Thymic Factor Therapy, Tome 16, pp. 21-29. New York: Raven Press.

Berthou, J., Migliore-Samour, D., LifChitz, A., Delettré, J., Floc'H, F. \& Jollès, P. (1987). FEBS Lett. 218, 55-58.

Cromer, D. T. \& ManN, J. B. (1968). Acta Cryst. A24, 321-324.

Delettré, J., Mornon, J. P. \& Lepicard, G. (1980). Acta Cryst. B36, 1430-1435.

Germain, G., Main, P. \& Woolfson, M. M. (1971). Acta Cryst. A27, 368-376.

IUPAC-IUB Commission on Biochemical Nomenclature (1970). Biochemistry, 9, 3471-3479.

Jollès, P., Parker, F., Floc'h, D., Migliore, D., Alliel, P., ZERIAL, A. \& WERNER, G. H. (1982). J. Immunopharmacol. 3, 363-369.

Migliore-S Amour, D., Bouchaudon, J., Floc'h, F., Zerial, A., Ninet, L., Werner, G. H. \& Jollès, P. (1980). Life Sci. 26, 883-888.

NaJjAR, V. \& FridKIN, M. (1983). Ann. NY Acad. Sci. 419, 1-273.

Parker, F., Migliore-Samour, D., Floc'h, F., Zerial, A., Werner, G. H., Jolles, J., Casaretto, M., ZahN, H. \& JOLLES, P. (1984). Eur. J. Biochem. 145, 677-682.

Prangé, T. \& Pascard, C. (1979). Acta Cryst. B35, 1812-1819.

Précigoux, G., Geoffre, S. \& Ouvrard, E. (1986). Acta Cryst. C42, 721-724.

Werner, G. H., Floc'h, F., Migliore-Samour, D. \& Jollès, P. (1986). Experientia, 42, 521-530. 